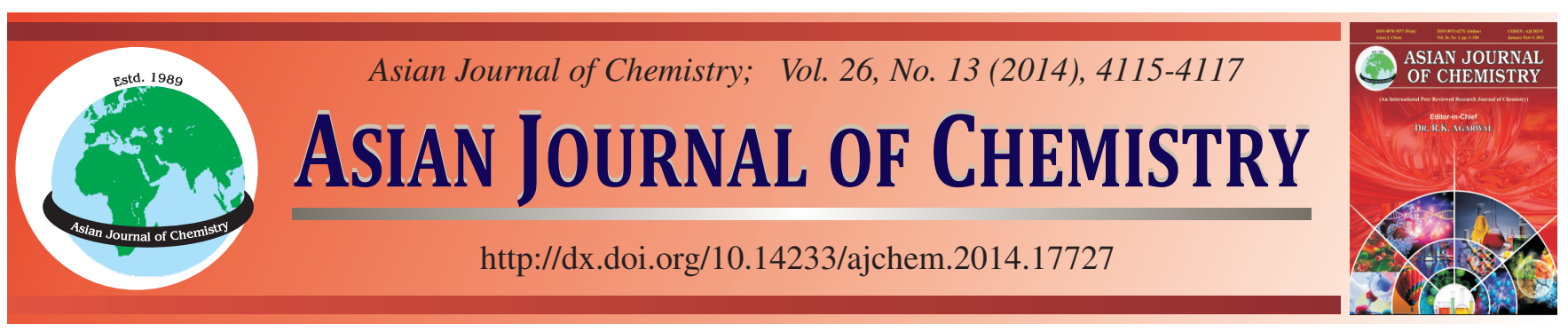

\title{
Ultrasonic Assisted Synthesis of Graphene Based PbSe Nanocomposite with Enhanced Photocatalytic Activity $\dagger$
}

\author{
Lei Zhu, Sun-Bok Jo, Shu Ye, Kefayat Ullah and Won-Chun OH*
}

Department of Advanced Materials Science \& Engineering, Hanseo University, Seosan-si, Chungnam-do 356-706, Republic of Korea

*Corresponding author: Fax: +82 41 6883352; Tel: +82 41 6601337; E-mail: we_oh@hanseo.ac.kr

In this article, we report a novel composite PbSe-graphene nanocomposite synthesis via facile ultrasonic assisted method. The structural and physicochemical properties of $\mathrm{PbSe}$-graphene composite are further characterized by X-ray diffraction, scanning electron microscopy with an energy dispersive X-ray analysis. The photocatalytic activity of PbSe-graphene was evaluated by measuring the photocatalytic degradation of organic pollutants such as methylene blue, industrial dyes such as Texbrite BA-L (TBA) under visible light. The photocatalytic degradation was analyzed using UV/visible spectrophotometer. The kinetic studies were also conducted to determine the order and the rate of the photocatalytic degradation of the organic dyes.

Keywords: Graphene, PbSe, Ultrasonics, Visible light, Photocatalytic activity.

ᄂ - - - - - - - - - - - - - - - - - - - - - - - - - - - - - - - - - -

\section{INTRODUCTION}

Semiconductor photocatalysis has been previously described as a promising option to remove persistent pollutants from contaminated water. Photocatalysis involves the in situ generation and use of highly oxidizing agents, mainly hydroxyl radicals. The hydroxyl radical possesses natural properties that enable it to degrade organic pollutants in water to obtain a complete mineralization into $\mathrm{CO}_{2}$, water and mineral acids such as sulfuric acid, hydrochloric acid and nitric acid. Among various semiconductor nanocrystals the small band gap of the $\mathrm{PbSe}^{1}$ makes it interesting for fundamental research and potential applications in photocatalysis.

Several approaches has been reported that graphene can be used as good support for semiconductor nanocrystals due to unique electronic, mechanical, physical and chemical properties $^{2-4}$. Flat monolayer structure and charge transferring ability of graphene make it suitable candidate to anchor $\mathrm{PbSe}$ nanocomposite and will help to improve the photocatalytic activity to overcome recombination process. Many approaches, such as hydrothermal methods ${ }^{5}$, sol-gel methods ${ }^{6}$, have been developed to decorate graphene sheets with nanoparticles. Ultrasonication is an effective technique for attachment of nanoparticles on grephene sheet. Ultrasonication involves the use of ultrasound as a source of high energy at a frequency range of $18-100 \mathrm{kHz}$ causing the creation of a phenomenon called acoustic cavitations. It involves the formation, growth and collapse of cavity bubbles that entraps dissolved gases or vapours surrounding water ${ }^{7,8}$. Ultrasonication has been found important in using for initiation or enhancement of catalytic reaction in both homogeneous and heterogeneous cases ${ }^{9}$.

In this manuscript $\mathrm{PbSe}$-graphene composite are prepared via a relatively facile ultrasoniction method. The objective of this paper is to experimentally prove that the photocatalytic degradative ability of $\mathrm{PbSe}$ decorated graphene composites is superlative in visible light using methylene blue and Texbrite BA-L.

\section{EXPERIMENTAL}

Synthesis of PbSe: For synthesis of PbSe precursor $2 \mathrm{~g}$ of anhydrous sodium sulfite $\left(\mathrm{Na}_{2} \mathrm{SO}_{3}\right)$ and $0.2 \mathrm{~g}$ of crude selenium powder $(\mathrm{Se})$ were vigourously stirred in $40 \mathrm{~mL}$ of distilled water for $0.5 \mathrm{~h}$ to ensure the homogenous mixing. Followed by addition of lead chloride $\left(\mathrm{PbCl}_{2}\right)$ suspension in 20 $\mathrm{mL}$ water. The resulting reaction mixture was then ultasonicatd for $90 \mathrm{~min}$ at $35^{\circ} \mathrm{C}$ and allowed to cool at room tempertaure. The PbSe precipitates were obtained was filtered using $47 \mathrm{~mm}$ Whatman filter paper. The residue was washed several time with distilled water and dried in vacume oven at $90^{\circ} \mathrm{C}$ for $8 \mathrm{~h}$.

Synthesis of PbSe-graphene composites: PbSe-graphene nanocomposite were prepared with ultrasonication method. In this process, $1 \mathrm{~g}$ of graphene oxide was mixed with previously 
obtained $\mathrm{PbSe}(0.1 \mathrm{~mol})$ in $200 \mathrm{~mL}$ distilled water. The suspension was ultrasonicated for $3 \mathrm{~h}$ at $35{ }^{\circ} \mathrm{C}$ and cooled at room temperature. The resultant solution was filtered with whatman filter paper $(47 \mathrm{~mm})$ and washed 3 times with distilled water and transferred into a dry oven for $6 \mathrm{~h}$ at $90^{\circ} \mathrm{C}$. The powder was then heat treated at $600{ }^{\circ} \mathrm{C}$ for $1 \mathrm{~h}$ in electric furnace to obtain 0.1 mol PbSe-graphene composites.

Photocatalytic studies: In order to analysis of the photocatalytic effect, the degradation reaction of methylene blue and Texbrite BA-L in water was followed. Aqueous solution of $2 \times 10^{-5} \mathrm{M}$ of methylene blue and Texbrite BA-L was prepared with deionized water in a $100 \mathrm{~mL}$ flask. The samples were then irradiated with LED lamp (Fawoo, Lumidas-H) with visible light allowed to fall directly on the solutions from a distance of $90 \mathrm{~cm}$ through a hollow tube to isolate the reactor from other factors that might influence the experiment. The clean transparent solution was analyzed by UV-visible spectroscopy.

\section{RESULTS AND DISCUSSION}

Characterization: Fig. 1a is the illustration of deposition of PbSe on the GONS. Fig. 1b shows the XRD pattern of the product obtained from the above procedure. It can be seen that the diffractogram of graphene exhibits the typical peaks at 25.9 and $42.7^{\circ}$, corresponding to the graphite (002) and (100) reflections (Joint Committee for Powder Diffraction Studies (JCPDS) No. 01-0646), respectively. XRD pattern of $\mathrm{PbSe}$ nanomposites exhibit the characteristic (111), (220), (311), (222), (400), (331), (420), (422) and (511) reflections that correspond to the clausthalite crystal phase (JCPDS PDF: 00-065-0240) of lead selenide. However, no signal for any other phases about graphene oxide (001) or graphene (002) can be detected in $\mathrm{PbSe}$-graphene composite.

In Fig. 2a, it can be clearly seen that the pure graphene oxide sheets naturally aggregate and stack to multilayers with numerous edges. The morphology of the graphene observed is a flaky texture reflecting its layer structure. Agglomarted $\mathrm{PbSe}$ nanoparticles can be observed which were distributed on graphene sheets in Fig. 2b. The graphene sheets act like a bridge for $\mathrm{PbSe}$ nanoparticles which may be benificial to provide a path for the photogenerated electron and hence will enhance the photocatalytic performance.

To get information about change in elements and element weight $\%$, the prepared Graphene-PbSe composite were examined by EDX. Fig. 3 shows the EDX microanalysis and element weight \% of Graphene-PbSe composite. Main elements such as $\mathrm{C}, \mathrm{Pb}$ and $\mathrm{Se}$ are existed. The strong $\mathrm{C}$ signal should mainly originate from graphene nanosheets. A very low concentrations of impurities are present which may be due to experimental procedure. This confirms that PbSe-graphene hybrid has been synthesized successfully.

Photodecolorization process: Fig. 4(a) and (b) represent the degradation of methylene blue and Texbrite BA-L with Graphene-PbSe in visible light from which it is clear that the concentration of methylene blue and Texbrite BA-L gradually diminishing with increasing time for all of samples. Moreover, the dye solution increasingly lost its colour intensity as the dye concentration continued to decrease. The decrease in concentration was evaluated at the $\lambda_{\max }$ values of the dyes which were
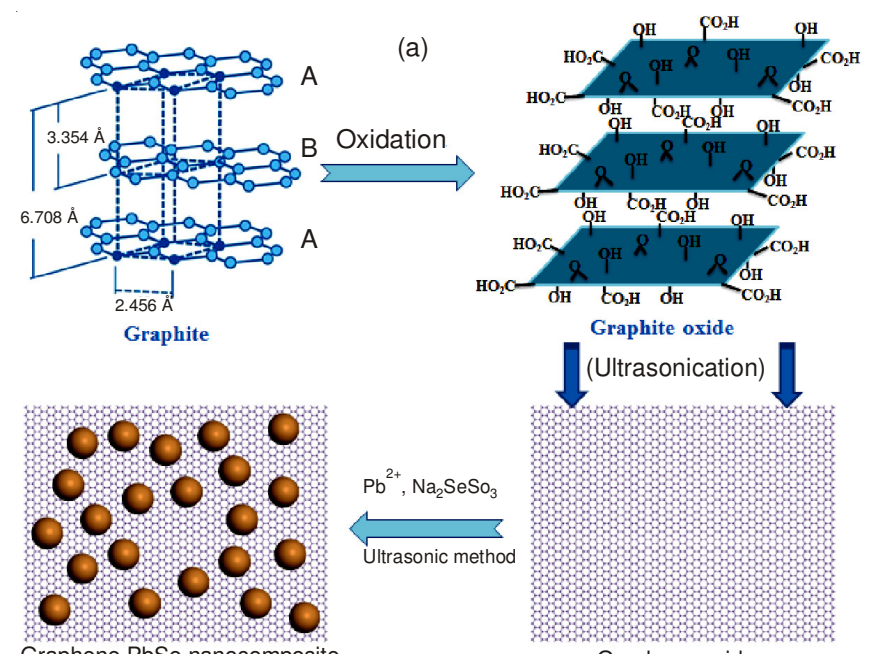

(Ultrasonication)

Graphene oxide

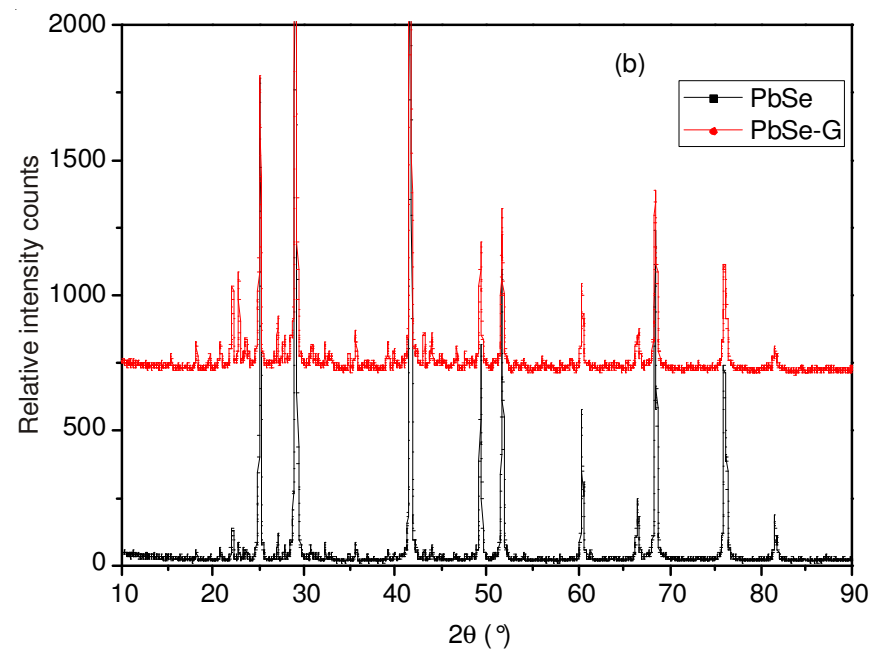

Fig. 1. (a) Schematic illustration of deposition of $\mathrm{PbSe}$ on the graphene, (b) XRD pattern of $\mathrm{PbSe}$ and graphene-PbSe composite
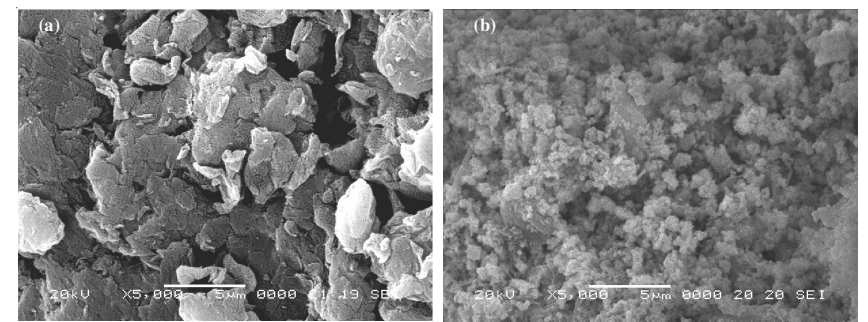

Fig. 2. SEM images of as-prepared composites: (a) graphene oxide, (b) graphene-PbSe composite
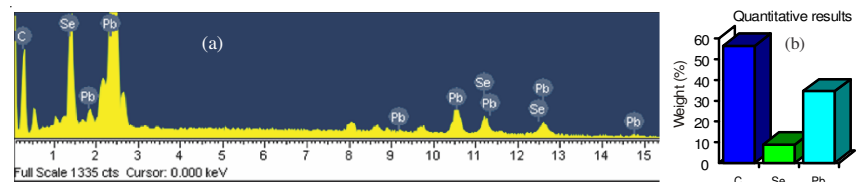

Fig. 3. EDX microanalysis (a) and element weight $\%$ (b) of graphene-PbSe composite

determined from the absorption spectra of the dyes. The $\lambda_{\max }$ values of methylene blue and Texbrite BA-L were found to be 665 and $265 \mathrm{~nm}$, respectively. The scheme of excitation and charge transfer process between PbSe particles and graphene nanosheets under visible light irradiation is shown in Fig. 5. 

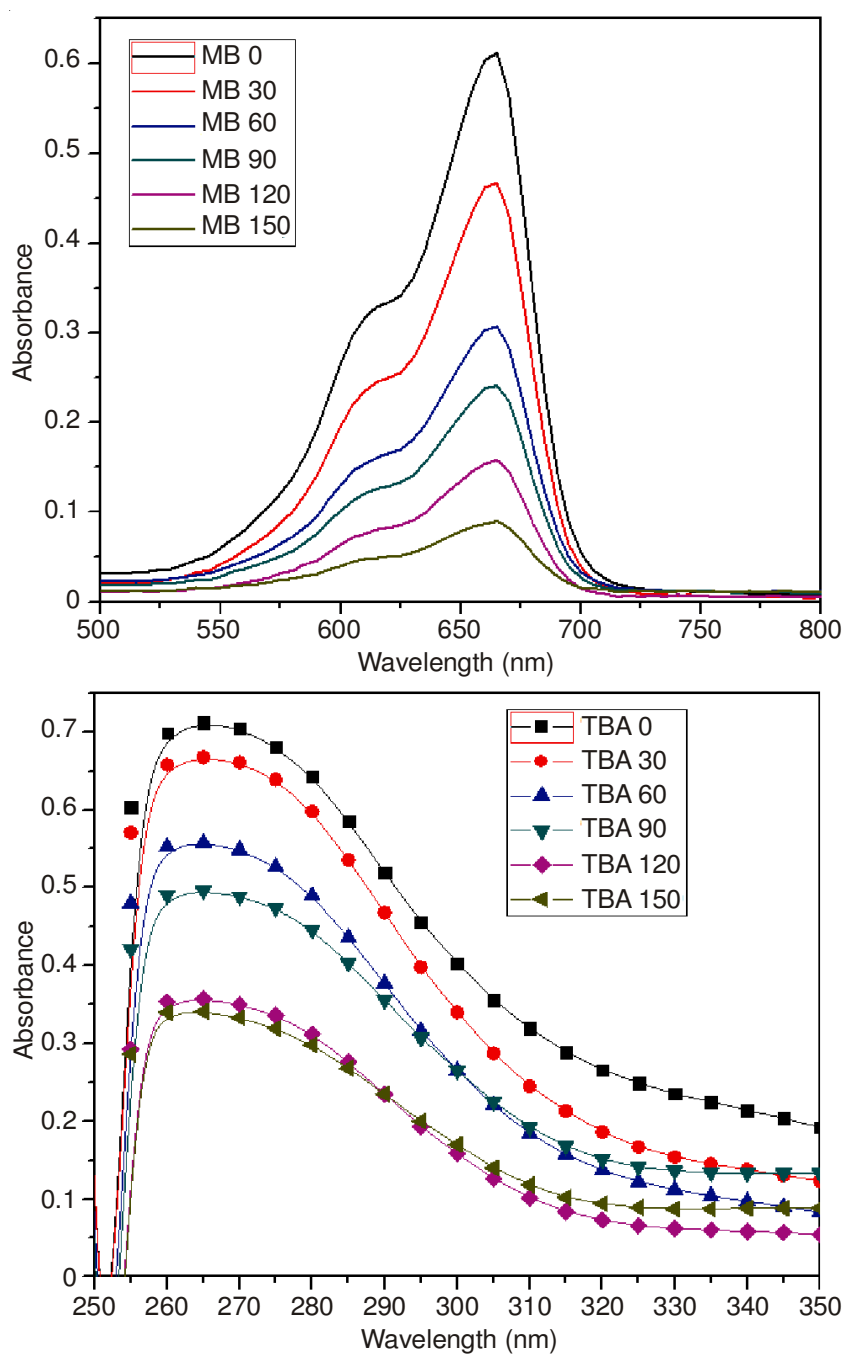

Fig. 4. Effect of the methylene blue and Texbrite BA-L decolorization process in presence of graphene-PbSe photocatalysts

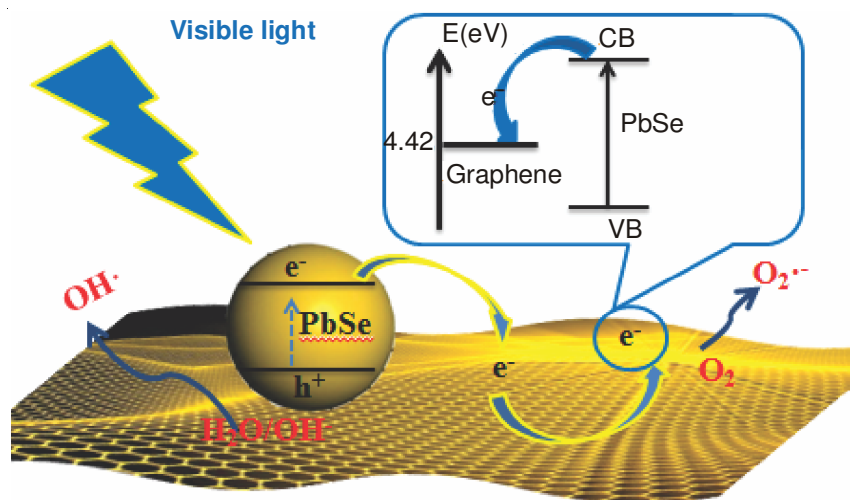

Fig. 5. Schematic diagram of the charge transfer between graphene and $\mathrm{PbSe}$ under visible light irradiation

\section{Conclusion}

In this study, PbSe-graphene composite photocataysts were prepared by ultrasonication method. XRD pattern of the $\mathrm{PbSe}$-graphene composite suggests the $\mathrm{PbSe}$ particles with clasthalite morphology. SEM images shows that PbSe nanoparticles were attached on graphene sheet. From the EDX data, the main elements like $\mathrm{Pb}, \mathrm{Se}, \mathrm{C}$ existed in the composites. The catalytic activity of $\mathrm{PbSe}$-graphene composites were examined by degradation of methylene blue and Texbrite BA-L in aqueous solutions under visible light irradiation. These results reveals the exceptional feature of graphene that make it an excellent supporting material for semiconductor nanoparticles as an electron acceptor and transporter.

\section{REFERENCES}

1. X.L. Li, X.R. Wang, L. Zhang, S.W. Lee and H.J. Dai, Science, 319, 1229 (2008).

2. C. Stampfer, E. Schurtenberger, F. Molitor, J. Guttinger, T. Ihn and K. Ensslin, Nano Lett., 8, 2378 (2008).

3. K. Haubner, J. Murawski, P. Olk, L.M. Eng, C. Ziegler, B. Adolphi and E. Jaehne, Chem. Phys. Chem., 11, 2131 (2010).

4. F. Lupo, R. Kamalakaran, C. Scheu, N. Grobert and M. Rühle, Carbon, 42, 1995 (2004).

5. X.Y. Zhang, H.P. Li, X.L. Cui and Y.H. Lin, J. Mater. Chem., 20, 2801 (2010).

6. A.Z. Abdullah and P.Y. Ling, J. Hazard. Mater., 173, 159 (2010).

7. N.H. Ince and G. Tezcanli-Güyer, Ultrasonics, 42, 591 (2004).

8. G.A. Tai and W.L. Guo, Ultrason. Sonochem., 15, 350 (2008).

9. T. Ghosh, K.Y. Cho, K. Ullah, V. Nikam, C.Y. Park, Z.D. Meng and W.C. Oh, J. Ind. Eng. Chem., 19, 797 (2013). 